AGRICULTURE AND BIOLOGY JOURNAL OF NORTH AMERICA

ISSN Print: 2151-7517, ISSN Online: 2151-7525, doi:10.5251/abjna.2011.2.8.1166.1170

(C) 2011, ScienceHuß, http://www.scihub.org/ABJNA

\title{
Study of yeast flora from fruit of Syzygium cumini (linn) skeel
}

\author{
Swapan kr. Ghosh \\ Mycopathology Laboratory ,Department of Botany , Ramakrishna Mission Vivekananda \\ Centenary College, Rahara, Kolkata -700118,West Bengal \\ swapan.krghosh@yahoo.com
}

\begin{abstract}
On the basis of morphological and biochemical characteristics five (5) genera and six (6) species of yeast from fruit surface of Jamun (Syzygium cumini (Linn.) Skeel ; Family-Myrtaceae ) from different zones of 24- Parganas $(\mathrm{N})$ districts of West Bengal ,India were isolated and identified .The identified yeasts were Candida famata, Candida ipomoeae ,Candida succiphila, Rhodototula mucilaginosa, ,Debaryamyces hansensii, Kodamaea anthophila and,Pichia lachancei,. There is no report of isolation of these yeast from this fruit from India till now .. Therefore, this fruit is good habitat for these speces of yeasts.
\end{abstract}

Key words: Yeast, Syzygium cumini, fruit,24 Parganas (N)

\section{INTRODUCTION}

Yeasts are unicellular eukaryotic fungi. The precise classification is a field that uses the characteristics of the cell, ascospore and colony. Physiological characteristics are also used to identify species. Yeasts are generally three types :a)Ascomycetous b)Basidiomycetous c)Deuteromycetous

Dimorphic yeasts are also present which become filamentous under certain environmental conditions. Ascomycetous are two types: Budding yeasts (Saccharomyces cerevisiae) and fission yeasts (Schizosaccharomyces pombe).

They are generally present in the natural resources .e i. leaf surface, fruit juice, palm syrup, toddy, milk, soil, animal surfaces and in the intestinal tracts of warm-blooded animals, where they may live symbiotically or as parasites etc. The common "yeast infection" is typically Candidiasis is caused by the yeast- Candida albicans. In addition to being the causative agent in vaginal yeast infections .Candida is also a cause of diaper rash and thrush of the mouth and throat.

From the ancient times yeast particularly Saccharomyces cerevisiae has been used in wine or alcohol making industries . in mordern sciences their uses are multifaceted :i) in the production of SCP ,ii) baking industries, iii)ethanol and acetic acid production, iv) biocontrol agents against many post harvest vegetable \& fruit diseases ,v) recombinant vaccine production and understanding of cell cycle process and so on
Therefore, their diversiy in natural sources is very much necessary .According to Kurtzman\& Fell (20062 there are about 100 genera and 700 species In some countries systemic study of yeasts flora from natural sources has been completed but India has vast natural sources for yeasts, its study of yeast flora is not done by any scientist.

The main objective of this experiment is to isolate different yeasts from fruit surfaces of jamun (Syzygium cumini ) ii) morphological ,microscopical and biochemical or physiological characterization of isolated yeast and to note their diversity on this fruit .

\section{MATERIALS AND MATHODS}

Site Description: The 24-Parganas(N) district of West Bengal is situated in the tropical zone and extends from latitude $22^{\circ} 11^{\prime} 6^{\prime \prime}$ north to $23^{\circ} 15^{\prime}$ $2^{\prime \prime}$ 'north and from longitude $88^{\circ} 20^{\prime}$ east to $89^{\circ} 5^{\prime}$ east .It is bordered to Nadia by north,to Bangladesh(Kulna Division by north and east, to south 24 -Parganas and Kolkata by south and to Kolkata, Howrah and Hooghly by west. The elevation of the district is $2,134 \mathrm{~m}$ (7001ft). It lies with in the Ganga- Brahmaputra delta ..The river Ganga flows along the entire west border of the district. There are many rivers ,such as Ichaamoti, Jamuna and Bidyadhuri. The soil status varies from alluvial to clay loam( wapedia.mobi/en/north _ 24_parganas). The study area includes mango orchard, bamboo bushes, ponds and the local vegetation which act like green lungs of the settlements and it also has some industries. According to the state of Forest Report (2003) ,the forest of the district has three kinds of forest $-\mathrm{i}$ ) very dense forest (16 sq.km ) ii) 
Moderately dense forest (36 sq $\mathrm{km}$ and open forest (75 sq km ). The hall mark is monsoon, which lasts from June to August. Annual rainfall is $1579 \mathrm{~mm}$. The weather remains humid and dry during the most time of the year except the rainy seasons. It remains dry during the winter (mid November to mid February) and humid during Summer ..Temperature remains $41^{\circ} \mathrm{C}$ (maximum) in May and $10^{\circ} \mathrm{C}$ (minimum) in January . Relative humidity remains between $50 \%$ in March and $90 \%$ in July.(http://famillpedia.wikia.com/wiki/north 24 p argana district\#climate ;State of Forest Report 2003)

Isolation \& Purification: Twenty fruit samples of Syzygium cumini (Linn)Skeel from different zone of 24-parganas $(N)$ of West Bengal ,India were collected in separate sterile biodegradable polythene bags and brought in laboratory .Known weight of each sample was washed with known volume of sterilized distilled water by shaking on a rotary shaker. The washings were 10 fold serially diluted and plated on MA medium ( $\mathrm{MA}$; malt extract, 2g;agar2g ;Distilled water, $100 \mathrm{ml}$ ) The Petri dishes were incubated at $25^{\circ}$ C for 3 days .

Single cell isolate of each type of yeasts was obtained by streaking loopfull of cells on MA medium and transferring well isolated colonies to MA. The isolates were maintained on Malt-Yeast -GlucosePeptone -Agar medium (Dry malt extract ,3g.;dry years ,3g.Peptone,5g.,D- glucose 10g, agar ,20g. Distilled water, $1 \mathrm{~L})$ of Wickerham $(1951)$ at $28^{\circ} \mathrm{C}$ with monthly subculturing.

Identification: Identificaton of each isolate of yeast up to species level was carried on the basis of standard morphological, and physiological /biochemical tests presented by Barnett et.al(2000)and Kurtzman \& Fell(2006) and Rose \& Harisson (1987-93)

Morphological \& Microscopical investigation: The colonies were observed and described on MA and MYGPA medium The isolates were also grown in MA \&MYPGA broth for determination of their cultural characteristics (pellicle ,sedimentation or ring formation,colour of colony, elevation of colony , texture of colony and shape of colony )). Under microscope, morphology (shape, size , number of ascospore, number of basidiospore, budding , pseudomycelium or mycelium ,etc ) of each cell of each yeast were recorded ( Ciupeanu,D,2008 ; Savova and Nikolova 2000-2003 )).In certain cases, the isolates were grown on sterile slices of carrot for induction of ascospore formation .

Phsiological or biochemical tests: For carbon and nitrogen assimilation, the basal medium of Barnett et.al(2000) will be used .The results will be determined after the, 3th, 7th, 14th, 21th and 28th day. The ability of some carbohydrates for anaerobic assimilation (fermentation) will be determined by using Durhan glass tubes after 3 weeks. . The quantity of the tested carbohydrates was $2 \%$.

For Diazonium blue -B (DBB) test, a ten day old culture on MYPGA will be held at 550 for the hours and then flooded with ice -cold DBB reagent The reagent will be prepared by dissolving diazonium blue salt (Sigma) in cold 0.5M-tris -HCL buffer pH 7.0 at $1 \mathrm{mg} / \mathrm{ml}$ (Vander Walt and. Hapsu -Havu 1976). The reagent will be kept ice -cold and used within few minutes of preparation.

Other additional tests such as starch formation ,urea hydrolysis, cycloheximide $(0.01 \%$ or $0.1 \%)$, will be performed.

The isolated yeasts will be stored by freeze-drying with protecting medium (Sucrose, 10\%, gelatine,1.5\% and agar $0.1 \%$ ).

\section{RESUSTS AND DISCUSSION}

The ,data presented in the tables 1-7, comparing with the standard keys of yeasts (Barnett et al. 2000, Kurtzman and Fell 2006 and Mushtaq et al. 2004)indicate that out of 13 isolates, $3\left(I_{1} I_{3} \& I_{7}\right)$ belong to the genus Candida sp. , $3\left(\mathrm{I}_{2}, \mathrm{I}_{4}, \& \mathrm{I}_{8}\right)$ belong to Rhodotorula sp. , $3\left(\mathrm{I}_{5}, \mathrm{I}_{9} \& \mathrm{I}_{13}\right)$ to Debaryomyces sp. and $2\left(I_{6} \& I_{10}\right)$ to Pichia sp. and remaining $2\left(\mathrm{I}_{11} \&\right.$ $\mathrm{I}_{12}$ ) are Kodamaea $\mathrm{sp}$. . Moreover $\mathrm{I}_{1}$ was Candida succiphila J.. D., Lee \& Komagata, $I_{3}$ is Candida ipomaeLachance et. al and $I_{7}$ was Candida famata . All 3 isolates of Rhodotorula were $R$. mucilaginosa The species of Pichia was P. lachancei Paff et. al. The Kodamaea $\left(I_{11} \& I_{12}\right)$ was $K$. anthophila Rosa et. al , All isolates of Debaryomyces were same i.e. $D$. hansenii(Zopf)Lodder \& Kreger -van Rij 
Table 1. Morphological \& microscopical characteristics of isolates of yeasts

\begin{tabular}{|c|c|c|c|c|c|c|c|c|c|c|c|c|c|}
\hline $\begin{array}{l}\text { Characteristic } \\
\mathrm{s}\end{array}$ & $\mathrm{I}_{1}$ & $\mathrm{I}_{2}$ & $I_{3}$ & $\mathrm{I}_{4}$ & $\mathrm{I}_{5}$ & $I_{6}$ & $I_{7}$ & $I_{8}$ & $I_{9}$ & $I_{10}$ & $I_{11}$ & $I_{12}$ & $I_{! 3}$ \\
\hline Colour & Wh & Red & Wh & Red & $\begin{array}{l}\text { Wh. } \\
\text { /cr }\end{array}$ & $\mathrm{w} / \mathrm{cr}$ & Wh & Red & $\begin{array}{l}\text { Wh. } \\
\text { /cr }\end{array}$ & $\begin{array}{l}\text { wh/ } \\
\mathrm{cr}\end{array}$ & $\mathrm{w} / \mathrm{cr}$ & $\mathrm{wh} / \mathrm{cr}$ & Wh. /c \\
\hline Surface & Rou & $\mathrm{Sm}$ & Rou & $\mathrm{Sm}$ & Wrin. & $\mathrm{Sm}$ & Rou & Rou & Wrin & $\mathrm{Sm}$ & $\mathrm{Sm}$ & $\mathrm{Sm}$ & Wrin \\
\hline Margin & En & En & undu & en & undu & En & undu & en & undu & En & en & En & undu \\
\hline Elevetion & Sl.co & Co & co & co & SI. Co. & Co & co & $\mathrm{co}$ & SI. Co & Co & slco & Co & $\begin{array}{l}\text { Sl. } \\
\text { Con }\end{array}$ \\
\hline Cell- shape & Ellip & R/Elip & Sph & $\mathrm{R}$. & R/o. & $\mathrm{R} / \mathrm{o}$ & Ellip & $\mathrm{R}$ & $\mathrm{R} / \mathrm{o}$ & $\mathrm{R} / \mathrm{o}$ & $\mathrm{O}$ & $\mathrm{O}$ & $\mathrm{R} / \mathrm{o}$ \\
\hline Ascospore & $A$ & $A$ & A & $A$ & $P$ & $P$ & $A$ & $A$ & $\mathrm{P}$ & $P$ & $P$ & $P$ & $P$ \\
\hline Ballistispore & $A$ & $A$ & A & $A$ & A & $\mathrm{A}$ & A & $A$ & $A$ & A & $A$ & $\mathrm{~A}$ & A \\
\hline $\begin{array}{l}\text { Pseudo- } \\
\text { mycelium }\end{array}$ & $A$ & $\mathrm{P}$ & $P$ & $P$ & A & A & A & $P$ & $\mathrm{~A}$ & A & $A$ & $A$ & A \\
\hline True mycelium & $A$ & $A$ & A & $A$ & A & $A$ & A & $A$ & $A$ & A & $P$ & $P$ & A \\
\hline
\end{tabular}

Wh=White; Wh/cr= White/cream ; ;Rou.=Rough ;Sm.=Smooth; Wrin.=Wrinkled;

En.=Entire ;Undu= Undulating; SI. Co= Slightly convex; Co.=Convex; Ellip.=Ellipsoid ;Sph= Spherical ;R.=Round ;O.=Oval; A=Absent; $\mathrm{P}=$ Present

Table 2. Biochemical characteristics of different yeast isolates using carbon assimilating tests

\begin{tabular}{|c|c|c|c|c|c|c|c|c|c|c|c|c|c|c|}
\hline $\begin{array}{l}\text { Sr. } \\
\text { No. }\end{array}$ & $\begin{array}{l}\text { Carbon } \\
\text { assimilation test }\end{array}$ & $I_{1}$ & $\mathrm{I}_{2}$ & $\mathrm{I}_{3}$ & $\mathrm{I}_{4}$ & $I_{5}$ & $I_{6}$ & $I_{7}$ & $I_{8}$ & $I_{9}$ & $\mathrm{I}_{10}$ & $I_{11}$ & $\mathrm{I}_{12}$ & $I_{! 3}$ \\
\hline 1 & D-glucose & + & + & + & + & + & + & + & + & + & + & + & + & + \\
\hline 2 & D-galactose & + & + & + & + & + & - & + & + & + & - & + & + & + \\
\hline 3 & L-sorbose & + & + & + & + & $+/-$ & - & + & + & $+/-$ & - & + & + & $+/-$ \\
\hline 4 & D-xylose & + & + & + & + & $-/+$ & + & + & + & $-1+$ & + & $\mathrm{W}$ & $\mathrm{W}$ & $+/-$ \\
\hline 5 & D-arabinose & $\mathrm{N}$ & + & - & + & $-/+$ & - & Dw & + & $-1+$ & - & - & - & $-/+$ \\
\hline 6. & L-ramnose & $+/-$ & - & - & - & w & - & - & - & W & - & - & - & VW \\
\hline 7. & Sucrose & + & + & + & + & + & + & + & + & + & + & + & + & + \\
\hline 8. & Maltose & VW & + & + & + & + & + & + & + & + & + & + & + & + \\
\hline 9. & $\alpha, \alpha$ Trehalose & + & + & + & + & + & - & + & + & + & - & + & + & + \\
\hline 10. & Cellobiose & + & + & + & - & + & + & + & + & + & + & + & + & + \\
\hline 11. & Lactose & $\mathrm{N}$ & - & - & - & - & - & + & - & - & - & - & & - \\
\hline 12. & Raffinose & - & + & - & - & + & + & + & + & + & + & + & + & + \\
\hline 13. & Inulin & $\mathrm{N}$ & - & - & - & $\mathrm{V}$ & - & + & - & $\mathrm{V}$ & - & - & - & Vw \\
\hline 14. & Starch & $\mathrm{N}$ & - & - & + & - & - & - & - & - & - & - & & + \\
\hline 15. & Glycerol & $\mathrm{N}$ & + & W & + & + & + & + & + & + & + & W & W & + \\
\hline 16. & D-glucitol & $\mathrm{N}$ & W & + & W & - & & + & W & - & $\mathrm{N}$ & + & + & - \\
\hline 17. & D-mannitol & + & DW & + & DW & + & & + & DW & + & $\mathrm{N}$ & + & + & + \\
\hline 18. & Myo-inositol & - & - & - & - & - & - & - & - & - & - & - & - & - \\
\hline 19. & $\begin{array}{l}\text { 2-Keto-D- } \\
\text { glucose }\end{array}$ & + & + & + & + & + & + & - & + & + & + & $\mathrm{N}$ & $\mathrm{N}$ & + \\
\hline 20. & D-Glucuronate & $\mathrm{N}$ & - & $\mathrm{N}$ & - & $\mathrm{N}$ & $\mathrm{N}$ & + & - & $\mathrm{N}$ & $\mathrm{N}$ & + & + & $\mathrm{N}$ \\
\hline 21. & Succinate & $\mathrm{N}$ & + & W & + & $-(+)$ & + & - & + & $-(+)$ & + & + & + & $+/-$ \\
\hline 22. & Citrate & + & + & - & + & + & + & - & + & + & + & + & + & + \\
\hline 23. & Methanol & + & - & - & - & - & & - & - & - & & - & & - \\
\hline 24. & Ethanol & + & - & - & - & $\mathrm{N}$ & + & $d$ & - & $\mathrm{N}$ & + & W & W & $\mathrm{N}$ \\
\hline 25. & $\begin{array}{l}\text { Propane 1,2- } \\
\text { diol }\end{array}$ & $\mathrm{N}$ & $\mathrm{N}$ & $\mathrm{N}$ & $\mathrm{N}$ & $-(+)$ & $\mathrm{N}$ & $\mathrm{N}$ & $\mathrm{N}$ & $-1+$ & $\mathrm{N}$ & - & - & $+/-$ \\
\hline 26. & Butane 2,3-diol & $\mathrm{N}$ & $\mathrm{N}$ & $\mathrm{n}$ & $\mathrm{N}$ & - & $\mathrm{N}$ & $\mathrm{N}$ & $\mathrm{N}$ & $\mathrm{N}$ & $\mathrm{N}$ & - & - & $\mathrm{N}$ \\
\hline
\end{tabular}

Responses:+=Posative ;-= Negative ;+(-)=mostly positive with some negative ;- (+)=Mostly negative but withsome positive $; \mathrm{W}=$ weak $; \mathrm{w} /$-weak or negative; $\mathrm{W} /+=$ weak or positive $\mathrm{N}=$ Not determined; $\mathrm{D}=$ Delayed growth ; $\mathrm{WW}=\mathrm{Very}$ weak ; 
Table 3. Biochemical characteristics of different yeast isolates using nitrogen assimilating tests

\begin{tabular}{|l|l|l|l|l|l|l|l|l|l|l|l|l|l|l|}
\hline $\begin{array}{l}\text { Sr. No \& } \\
\text { Nitrogen } \\
\text { source. }\end{array}$ & $\mathrm{I}_{1}$ & $\mathrm{I}_{2}$ & $\mathrm{I}_{3}$ & $\mathrm{I}_{4}$ & $\mathrm{I}_{5}$ & $\mathrm{I}_{6}$ & $\mathrm{I}_{7}$ & $\mathrm{I}_{8}$ & $\mathrm{I}_{9}$ & $\mathrm{I}_{10}$ & $\mathrm{I}_{11}$ & $\mathrm{I}_{12}$ & $\mathrm{I}_{! 3}$ & \\
\hline 1. Nitrate & $\mathrm{W} /-$ & - & - & - & - & - & - & - & - & - & - & - & - & \\
\hline 2. Nitrite & + & - & - & - & $\mathrm{N}$ & - & $\mathrm{D}, \mathrm{W}$ & - & $\mathrm{N}$ & - & - & - & $\mathrm{N}$ & \\
\hline 3. L-Lysine & - & - & + & - & + & + & $\mathrm{D}, \mathrm{W}$ & - & + & + & + & + & + & \\
\hline 4. Cadaverine & $\mathrm{N}$ & - & + & - & + & + & $\mathrm{N}$ & - & + & + & + & + & + & \\
\hline 5. Glucosamine & $\mathrm{N}$ & - & - & - & $\mathrm{V}$ & - & $\mathrm{N}$ & - & $\mathrm{V}$ & - & $\mathrm{W}$ & $\mathrm{VW}$ & $\mathrm{W}$ & \\
& & & & & & & & & & & & & & \\
\hline
\end{tabular}

Table 4. Biochemical characteristics of different yeast isolates by fermentation tests

\begin{tabular}{|l|l|l|l|l|l|l|l|l|l|l|l|l|l|l|}
\hline Sr.No. & $\begin{array}{l}\text { Fermentation in } \\
\text { carbon source }\end{array}$ & $\mathrm{I}_{1}$ & $\mathrm{I}_{2}$ & $\mathrm{I}_{3}$ & $\mathrm{I}_{4}$ & $\mathrm{I}_{5}$ & $\mathrm{I}_{6}$ & $\mathrm{I}_{7}$ & $\mathrm{I}_{8}$ & $\mathrm{I}_{9}$ & $\mathrm{I}_{10}$ & $\mathrm{I}_{11}$ & $\mathrm{I}_{12}$ & $\mathrm{I}_{13}$ \\
\hline 1. & D-glucose & + & - & $\mathrm{W}$ & - & - & + & $\mathrm{W}$ & - & - & + & + & + & - \\
\hline 2. & D-galactose & $+(-)$ & - & $+/-$ & - & + & $\mathrm{W}$ & - & - & + & $\mathrm{VW}$ & $+/-$ & $+/-$ & + \\
\hline 4. & Sucrose & $\mathrm{N}$ & - & $\mathrm{N}$ & - & - & + & - & - & - & + & - & - & - \\
\hline 5. & Maltose & - & - & - & - & $\mathrm{VW}$ & $\mathrm{N}$ & + & - & $\mathrm{V}$ & $\mathrm{N}$ & $\mathrm{VW}$ & $\mathrm{VW}$ & $\mathrm{W}$ \\
\hline 6. & Lactose & - & - & - & - & - & $\mathrm{N}$ & - & - & - & $\mathrm{N}$ & - & - & - \\
\hline 7. & Inulin & $\mathrm{N}$ & - & $\mathrm{N}$ & - & - & $\mathrm{N}$ & $\mathrm{N}$ & - & - & $\mathrm{N}$ & - & - & - \\
\hline
\end{tabular}

Table 5. The growth of different yeast isolates without vitamine(s)

\begin{tabular}{|l|l|l|l|l|l|l|l|l|l|l|l|l|l|l|}
\hline Sr.No. & $\begin{array}{l}\text { Growth } \\
\text { without } \\
\text { vitamine }\end{array}$ & $\mathrm{I}_{1}$ & $\mathrm{I}_{2}$ & $\mathrm{I}_{3}$ & $\mathrm{I}_{4}$ & $\mathrm{I}_{5}$ & $\mathrm{I}_{6}$ & $\mathrm{I}_{7}$ & $\mathrm{I}_{8}$ & $\mathrm{I}_{9}$ & $\mathrm{I}_{10}$ & $\mathrm{I}_{11}$ & $\mathrm{I}_{12}$ & $\mathrm{I}_{13}$ \\
\hline 1 & $\begin{array}{l}\text { Without } \\
\text { vitamines }\end{array}$ & $\mathrm{V}$ & + & - & + & $+/-$ & - & + & + & $+/-$ & - & - & - & $+/-$ \\
\hline 2 & $\begin{array}{l}\text { Without myo- } \\
\text { inosital }\end{array}$ & $\mathrm{N}$ & + & $\mathrm{N}$ & + & + & + & $\mathrm{N}$ & + & + & + & + & + & + \\
\hline 3 & $\begin{array}{l}\text { Without } \\
\text { biotin }\end{array}$ & $\mathrm{N}$ & + & $\mathrm{N}$ & + & + & $+/-$ & $\mathrm{N}$ & + & + & $+/-$ & $\mathrm{V}$ & $\mathrm{V}$ & + \\
\hline 4 & $\begin{array}{l}\text { Without } \\
\text { thiamine }\end{array}$ & + & + & & + & + & & + & + & + & $\mathrm{V}$ & $+/-$ & $+/-$ & + \\
\hline 5 & $\begin{array}{l}\text { Without biotin } \\
\& \text { thiamine }\end{array}$ & $\mathrm{N}$ & + & $\mathrm{N}$ & + & $+/-$ & & $\mathrm{N}$ & + & $+/-$ & - & - & - & $+/-$ \\
\hline 6 & $\begin{array}{l}\text { Without } \\
\text { pyridoxine }\end{array}$ & $\mathrm{N}$ & + & $\mathrm{N}$ & + & $\mathrm{N}$ & & $\mathrm{N}$ & + & $\mathrm{N}$ & + & $\mathrm{N}$ & $\mathrm{N}$ & $\mathrm{N}$ \\
\hline 7 & $\begin{array}{l}\text { Without } \\
\text { niacin }\end{array}$ & $\mathrm{N}$ & + & $\mathrm{N}$ & + & $\mathrm{N}$ & & $\mathrm{N}$ & + & $\mathrm{N}$ & + & $+/-$ & $\mathrm{V}$ & $\mathrm{N}$ \\
\hline
\end{tabular}

Table 6.The growth of different yeast isolates at different temperatures

\begin{tabular}{|l|l|l|l|l|l|l|l|l|l|l|l|l|l|l|}
\hline Sr.No. & $\begin{array}{l}\text { Growth at } \\
\text { temperature }\end{array}$ & $I_{1}$ & $I_{2}$ & $I_{3}$ & $I_{4}$ & $I_{5}$ & $I_{6}$ & $I_{7}$ & $I_{8}$ & $I_{9}$ & $I_{10}$ & $I_{11}$ & $I_{12}$ & $I_{! 3}$ \\
\hline 1 & $25^{\circ} \mathrm{C}$ & + & + & + & + & + & + & + & + & + & + & + & + & + \\
\hline 2 & $30^{\circ} \mathrm{C}$ & + & + & + & + & + & + & + & + & + & + & + & + & + \\
\hline 3 & $35^{\circ} \mathrm{C}$ & + & + & + & + & + & + & + & + & + & + & + & + & + \\
\hline 4 & $37^{\circ} \mathrm{C}$ & + & + & - & + & + & - & - & + & + & - & + & + & + \\
\hline 5 & $40^{\circ} \mathrm{C}$ & + & - & - & - & + & - & - & - & + & - & - & - & + \\
\hline
\end{tabular}


Table 7. Some additional tests for characterization of yeast isolates

\begin{tabular}{|l|l|l|l|l|l|l|l|l|l|l|l|l|l|l|}
\hline Sr.No. & test & $\mathrm{I}_{1}$ & $\mathrm{I}_{2}$ & $\mathrm{I}_{3}$ & $\mathrm{I}_{4}$ & $\mathrm{I}_{5}$ & $\mathrm{I}_{6}$ & $\mathrm{I}_{7}$ & $\mathrm{I}_{8}$ & $\mathrm{I}_{9}$ & $\mathrm{I}_{10}$ & $\mathrm{I}_{11}$ & $\mathrm{I}_{12}$ & $\mathrm{I}_{13}$ \\
\hline 1 & $\begin{array}{l}\text { Diazonium blue } \\
-\mathrm{B}\end{array}$ & $\mathrm{N}$ & + & - & + & - & - & - & + & - & - & - & - & - \\
\hline 2 & $\begin{array}{l}\text { Starch } \\
\text { formation }\end{array}$ & $\mathrm{N}$ & - & - & - & $\mathrm{N}$ & - & - & & $\mathrm{N}$ & - & - & - & $\mathrm{N}$ \\
\hline 3 & Urea hydrolysis & - & + & - & + & - & - & - & + & - & - & - & - & - \\
\hline 4 & $\begin{array}{l}\text { Cyclohexamide } \\
(.01 \%)\end{array}$ & $\mathrm{Vw}$ & & $\mathrm{VW}$ & & - & - & - & & - & - & - & - & - \\
\hline 5 & $\begin{array}{l}\text { Cyclohexamide } \\
(.0 .1 \%)\end{array}$ & - & & - & & - & - & - & & - & - & - & - & - \\
\hline
\end{tabular}

Rhodotorula mucilaginosa is anamorphic basidiomycota yeast, while,D. hansenii ,Kodamaea anthophila and Pichia lachancei are telomorphic ascomycota yeasts but Candida Sps are anamorphic yeasts. All species appeared newly reported from India. Moreover, their isolation from the fruit surface of Syzygium cumini appeared newly reported. .Candida $\mathrm{sp}$ and Rhodotorula $\mathrm{sp}$ were isolated and characterized from palm syrup, molasses ,toddy and grapes in India(Ghosh and Samadder,1991)

Candida ipomoeae, Kodamaea anthophila and Pichia lachancei were reported earlier from Ipomaea sp \& insect ,flower of Hibiscus $\mathrm{sp}$ and rotting bark respectively (Barnetts et. al 2000). Rhodotorula mucilaginosa was reported from Bulgarian milk product (Savova, and..Nikolova 2002)) .Candida succiphila $D$. hansenii ,C. famata were recorded by many workers(Barnett et. al 2000, Ciupeanu, 2000, Mushtaq et al. 2004,\& Savova, and..Nikolova 2002) in abroad from various natural sources

CONCLUSION AND RECOMMENDATIONS: Yeasts isolated from fruit of Syzygium cumini from 24Pargana (N)district West Bengal , India, belong to five(5) genera ; Candida (3 species), Pichia (1 sp ) Rhodotorula (! Species ), Debaryamyces (1 species)and Kodamaea (1 species). Expected this work will encourage to evaluate yeast flora from different natural sources that may include industrially useful yeast as well.

\section{ACKNOWLEDGEMENT}

Thanks are due to Prof. K.R.Samaddar Kalyani University , Nadia , W.B. India for helpful suggestions and also to the principal, Ramakrishna Mission
Vivekananda Centenary College, Kolkata700118, The author also acknowledges UGC ,New Delhi for financial help .

\section{REFERENCES}

Barnett ,I .A, P .W. Payee;and D. Yarrow 2000Yeasts: Characterization and Identification , $3^{\text {rd }}$ ed. Cambridge University Press, Cambridge.

Ciupeanu, D. 2000The Identification of Some Yeast Speces from the Banu Maracine Ecotope. Bulletin UASVM, Horticulture,65(1);350-355

Ghosh ,S.K., Samadder, K.R. 1991.Characterization and Biomass Production Potential of Yeast Flora of Some Natural Sources of Kalyani, J. Mycopathol. Res. 29(2):111-117

Kurtzman, C.P., Fell, J.W. 2006 "Yeast Systematics and Phylogeny Implications of Molecular Identification Methods for Studies in: Ecology.", Biodiversity and Ecophysiology of Yeasts, The Yeast Handbook, Springer

Mushtaq, M. ;S ,Nahar ;M.H .Hashmi 2004.Isolation and identification of Yeast Flora from Soil of Karachi, Pakistan .Pak. J. Bot. ,36(1):173-180

Rose, A. .H., J .S .Harrison, 1987-1993The Yeasts. Vol. !5 Academic Press London

Savova,I and. .Nikolova, M 2002 Isolatoin and Taxonomic Study of Yeast Strains from BulgarianDairy Produvts, Journal of Culture Collections, 3,(.1) : 59-65.

Vander Walt ,J.,P.;V. K. Hapsu -Havu 1976 A colour reaction for the identification ofascomycetous and basidiomycetous yeasts Anthoie van Leeuwenhoek. 42:157-163

Wickerham, L.J. 1951Taxonomy of Yeasts, Technical Bulletin No.1029 United States Department of Agriculture 